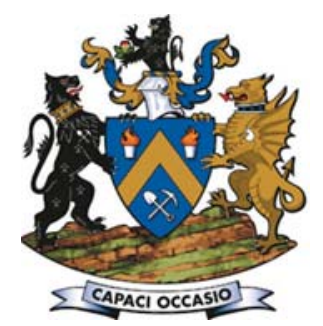

\title{
Effect of crushing on near-gravity material distribution in different size fractions of an Indian non-coking coal
}

\author{
by S. Mohanta*, B. Sahoo*, I.D. Behera*, and S. Pradhan*
}

\section{Synopsis}

Run-of-mine coal contains particles of different sizes with different specific gravities, and when this coal is crushed, the amount of material present in a particular density class changes. This alters the washability characteristics of the coal, which affects the quantity, quality, and separation efficiency of the gravity-based coal washing process. The degree of difficulty in washing a typical coal at a particular specific gravity depends mostly on the amount of material occurring within \pm 0.1 specific gravity range, which is known as 'near-gravity material' at that particular specific gravity of separation. In this paper, two numerical indices, namely 'near-gravity material index' and 'index of washability', are used to quantify the distribution of near-gravity material in different density classes and to evaluate the degree of difficulty involved in the washing process. The results of this preliminary investigation indicate the uniform distribution of near-gravity material in all density classes for finer size fractions, whereas for coarser size fractions more near-gravity material is present in the lower density classes, making the washing process more difficult at a lower specific gravity of separation.

Keywords

coal, coal cleaning, near-gravity material, index of washability.

\section{Introduction}

The prime objective of coal beneficiation is to remove physical impurities as effectively and economically as possible. The beneficiation process includes various operations on the asmined coal to make it more suitable for enduse application without destroying the physical identity of the coal. There are several coal beneficiation processes, but gravity-based separators remain the most efficient unit operation for removing the undesirable gangue material from run-of-mine coal. These separators exploit the difference in density between coal particles and gangue particles to effect a separation. The feed is introduced into an aqueous suspension of ultrafine magnetite with a particular density. The feed particles with a relative density less than that of the suspension (clean coal) float to the top surface of the suspension, whereas particles denser than the suspension density (gangue material) sink to the bottom. When the difference in density between the particle and the aqueous suspension is large, the separation is easy, but when this difference is less the separation becomes much more difficult since the settling velocity of the particles is very low. This lower settling velocity increases the probability that particles that should report to floats report to sinks, and vice versa, hence increasing the amount of misplaced material. Therefore, the degree of difficulty in beneficiating a typical coal at a particular specific gravity depends on the amount of material occurring within \pm 0.1 specific gravity range. This is known as 'neargravity' material, also termed 'near-dense' material, at that particular specific gravity of separation.

As coal is heterogeneous in nature, the density of a daughter coal particle depends on the nature of the parent coal particle from which it has been produced and the fracture patterns involved during crushing, as shown in Figure 1. As the fracturing of particulate material is a random process and many factors contribute to the fracturing of any single particle, the result is never exactly predictable. Particles of the same material and similar in size and shape show a wide variation in individual fracture patterns. Therefore, when a bigger heterogeneous particle of particular density is subjected to crushing a broad spectrum of particles of various sizes and densities is produced. Similarly, when run-ofmine coal is crushed, a wide variety of smaller particles are generated, which alters the amount of near-gravity material present in a particular density class and accordingly changes the degree of difficulty in washing. Therefore, it is imperative that the change in mass of the near-gravity material at a particular specific gravity on crushing should be determined accurately.

The difficulty in washing a particular coal can be interpreted from the steepness of the 'characteristic curve', which is derived from the sink-and-float data (Corriveau and

* Department of Chemical Engineering, Indira Gandhi Institute of Technology, Sarang, India.

(C) The Southern African Institute of Mining and Metallurgy, 2016. ISSN 2225-6253. Paper received Jul. 2015; revised paper received Oct. 2015. 


\section{Effect of crushing on near-gravity material distribution in different size fractions}

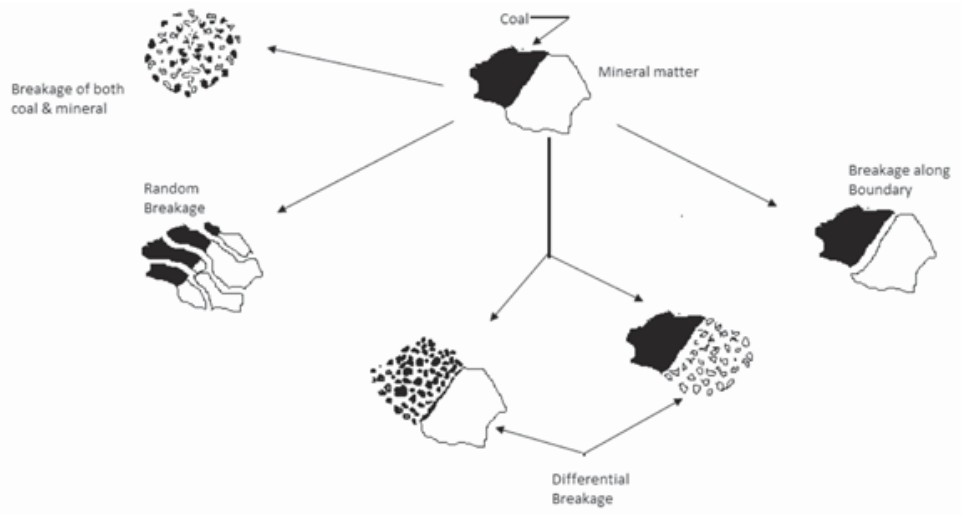

Figure 1-Breakage patterns of coal particles

Schapiro, 1979; Osborne, 1988; Salama, 1989; Salama and Mikhail, 1993). Also, several indices, coefficients, and factors such as the index of washability (Govindarajan and Rao, 1994), washability number (Sarkar et al., 1977), and washability index (Sarkar et al., 1962) have been proposed to quantify the amenability of a particular coal towards washing, but none of them has gained wide acceptance among the coal preparation fraternity because each of these parameters suffers from inherent limitations. In the present work, two simple parameters from the literature, namely 'near-gravity material index' (NGMI) and 'index of washability' (IW), are reviewed and utilized to evaluate the effect of crushing on the distribution of near-gravity material in different density classes. The primary reason for selecting the parameter IW for this study is that it varies between zero and 100 , thus facilitating quick interpretation, and provides an explicit equation and hence a precise evaluation of coal washability characteristics is possible. Similarly, the NGMI varies from zero to 1 for 'easy to wash' and 'difficult to wash' coal respectively, thus also facilitating rapid interpretation. Moreover, the NGMI generates more useful information from the sink-and-float data for a typical coal and provides an easier way to compare the washability characteristics of different coals quantitatively.

\section{Theoretical background}

Govindarajan and Rao (1994) considered the float fraction of the sink-and-float analysis as the recovery of two constituents, namely non-ash $\left(R_{N}\right)$ and ash-forming $(R A)$ materials, which can be calculated by the equations

$$
\begin{aligned}
R_{N} & =\frac{100 \cdot X \cdot\left(100-C_{A}\right)}{100-H_{A}} \\
R_{A} & =\frac{100 \cdot X \cdot C_{A}}{H_{A}}
\end{aligned}
$$

where $X$ is the cumulative fractional weight of the feed coal floated, and $C_{A}$ and $H_{A}$ are the ash percentages of the float fraction and feed, respectively. Also, they observed that a three-constant cubic equation of the following form is a good fit when the recovery curves for $R_{N}$ and $R_{A}$ are plotted against cumulative fractional weight.

$$
R_{N}=a X+b X^{2}+c X^{3}
$$

$$
R_{A}=p X+q X^{2}+r X^{3}
$$

In order to demonstrate the calculation procedure, the sink-and-float analysis results of $-150+25 \mathrm{~mm}$ size fraction of Ananta OCP coal is considered (Table I). The values for $R_{N}$ and $R_{A}$ are calculated from Equations [1] and [2] and are tabulated in Table I. The recovery curves for non-ash and ash-forming materials (curve $\mathrm{ADC}$ and curve $\mathrm{ABC}$ in Figure 2) are drawn by plotting $R_{N}$ and $R_{A}$ values against cumulative fractional weight. The values of the constants in Equations [3] and [4] are estimated by fitting these equations to the non-ash and ash recovery curves and by using the least squares method. In the present study a commercial software package named DataFit Version 8.0 is utilized to calculate the values for these constants. For floatand-sink data in Table I, the calculated values for $a, b, c, p$, $q$, and $r$ are $128.17,-4.76,-22.93,46.84,8.99$, and 43.26, respectively.

On considering the area bounded by these two curves for the recoveries of non-ash and ash-forming material, Govindarajan and Rao (1994) proposed the index of washability (IW). The final form of the equation is represented as

$$
I W=\frac{\{6 \mathrm{a}+4 \mathrm{~b}+3 \mathrm{c}-600\}\{6(\mathrm{a}-\mathrm{p})+4(\mathrm{~b}-\mathrm{q})+3(\mathrm{c}-\mathrm{r})\}}{3600 H_{A}}
$$

This index of washability varies between zero and 100 for the 'unwashable' and 'easiest washable' coal, respectively. For the data tabulated in Table I, the value of IW is found to be 15.259. The IW values for other size fractions of coal are calculated following the same procedure.

Based on the same concept, Majumder and Barnwal (2004) proposed the 'near-gravity material index' (NGMI) for a better comparison between the coals. Curves ADC and $\mathrm{ABC}$ of Figure 2 represent the recovery curves for non-ash and ash-forming materials, respectively. The amount of neargravity material (NGM) at a particular specific gravity is defined as the difference in cumulative fractional weights floated at \pm 0.1 specific gravity around that specific gravity. Using this definition with the data in Table I, the NGM at 1.45 specific gravity may be calculated from the difference between the cumulative fractional weight floated at 1.55 and the cumulative fractional weight floated at 1.35 (i.e. 0.737 $0.298=0.439$ ). In terms of recovery of non-ash and ashforming materials, the NGM distribution at 1.45 may be 


\section{Effect of crushing on near-gravity material distribution in different size fractions}

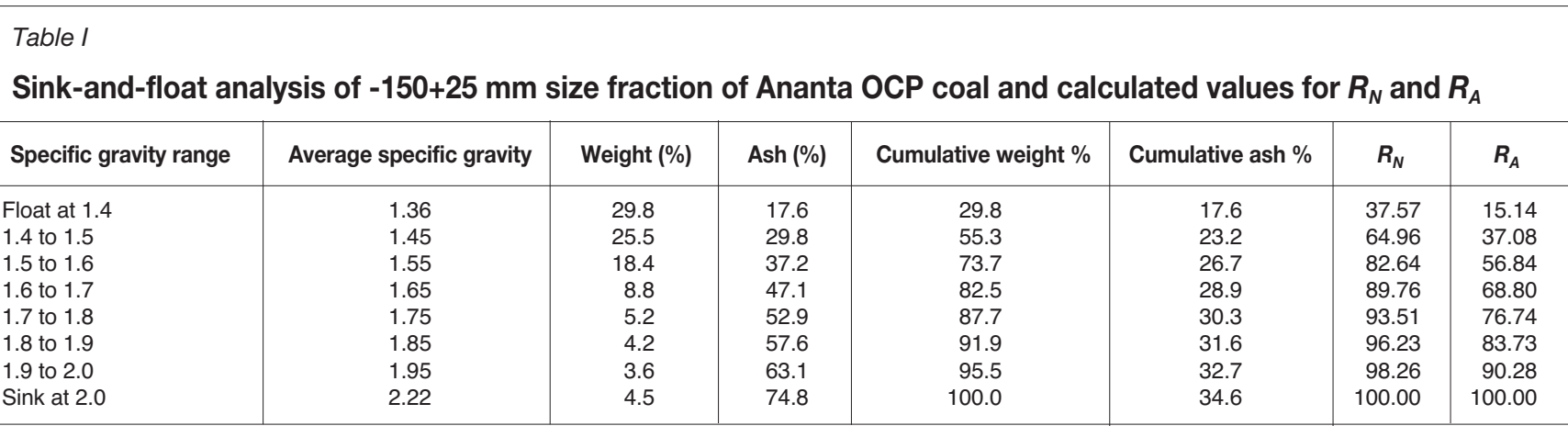

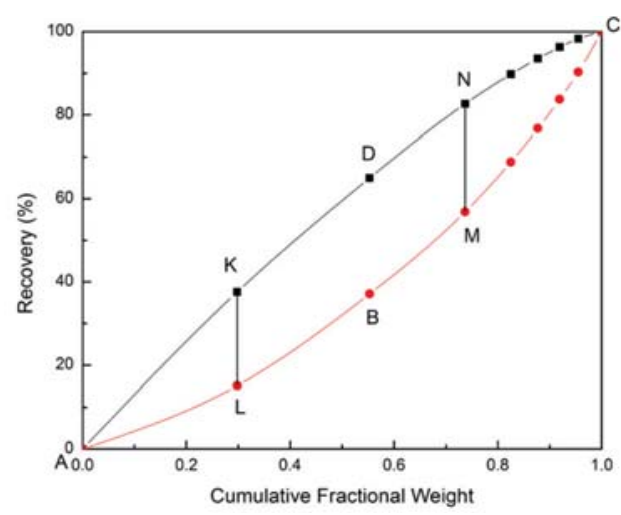

Figure 2-Recovery curves for $-150+25 \mathrm{~mm}$ size fraction of Ananta OCP coal

represented geometrically by the area under KLMN in Figure 2. This area will be different at different specific gravities of separation for a particular coal. The near-gravity material index can be calculated from the following equation:

$$
\begin{aligned}
\text { NGMI } & =\frac{\text { Area of KLMN }}{\text { Area between curves ADC and ABC }} \\
= & \frac{\text { Area under the curve KDN }- \text { Area under the curve LBM }}{\text { Area under the curve ADC }- \text { Area under the curve ABC }} \\
& =\frac{\int_{0.3}^{0.66}\left(a X+\mathrm{bX}^{2}+\mathrm{cX}^{3}\right) \mathrm{dX}-\int_{0.3}^{0.66}\left(\mathrm{pX}+\mathrm{qX}^{2}+\mathrm{rX}^{3}\right) \mathrm{dX}}{\int_{0}^{1}\left(\mathrm{aX}+\mathrm{bX}^{2}+\mathrm{cX}^{3}\right) \mathrm{dX}-\int_{0}^{1}\left(\mathrm{pX}+\mathrm{qX}^{2}+\mathrm{rX}^{3}\right) \mathrm{dX}}
\end{aligned}
$$

\section{Procedure adopted}

As per the test programme, a coal sample of $4 \mathrm{t}$ was collected (Indian Standard (IS):436, Part 1/Section 1) from Ananta $\mathrm{OCP}$, and three representative samples prepared after coning and quartering the collected sample. Each representative sample was individually crushed to $150 \mathrm{~mm}, 100 \mathrm{~mm}$, and $50 \mathrm{~mm}$ in a laboratory-type roll crusher and the crushed products subjected to screen analysis.

Sink-and-float tests (IS: 13810) were conducted for different size fractions. This test is conducted primarily to determine how much coal of what quality can be produced at a given specific gravity with what separation complexity (Mitchell and Charmbury, 1963). Liquids in a range of equally spaced specific gravity intervals from 1.4 to 2.0 are prepared, which include 1.4, 1.5, 1.6, 1.7, 1.8, 1.9, and 2.0. The liquids from density 1.4 to 1.6 are prepared from solutions of zinc chloride in water, and the rest by mixing carbon tetrachloride and bromoform in different proportions. The separation tank is part-filled with the required liquid, the relative density of which is then checked using a suitable hydrometer. The relative density of the liquid is adjusted to the correct reading and maintained at that value by frequent checking to ensure that it remains within the range of \pm 0.002 of the desired relative density for the duration of the test.

Each size fraction of coal obtained after screen analysis is introduced into the tank containing the liquid of density 1.4 and gently agitated. Care is taken not to overload the tank as this is liable to interfere with the separation of entrained near gravity material. After allowing sufficient time for separation, the float material is removed and collected on a draining platform. The settled sink material is agitated to release any entrained float material. Then, all the float materials are washed in water, air-dried, and weighed for further analysis. The sink material is well drained and care is taken to maintain the relative density of the succeeding liquid by introducing only completely drained material from the previous test. It is then introduced into the liquid of next higher relative density (density 1.5). The float material from this separation is also washed, air-dried, weighed, and prepared for further analysis. This procedure is repeated until all the coal fractions have been tested at all relative densities.

The ash content of each sink-and-float fraction is determined as per IS: 1350 . The ash content is determined by heating a weighed quantity of powdered coal $(<212 \mu \mathrm{m})$ in an open crucible in presence of air to $500^{\circ} \mathrm{C}$ in 30 minutes, from 500 to $815^{\circ} \mathrm{C}$ for further 60 minutes, and maintained at this temperature until a constant weight is achieved (approximately 60 minutes). The weight of residue is the ash content of the coal sample. A typical set of sink-and-float data for $-150+25 \mathrm{~mm}$ size fraction is shown in Table I for the representative coal sample crushed to $150 \mathrm{~mm}$.

\section{Results and discussion}

On utilizing the sink-and-float data, the recovery curves for all the size fractions were drawn for each of the three representative samples. Accordingly, the parameters for the cubic equation were calculated by fitting the equation to the data points and adopting the least squares approach. Using these parameters and methodologies described earlier, the NGMI values were calculated and plotted as a function of specific gravity of separation as shown in Figure 3. It is interesting to note that for all the three representative samples, the pattern of the curves is similar. The NGMI is higher at lower specific gravity of separation and decreases 


\section{Effect of crushing on near-gravity material distribution in different size fractions}

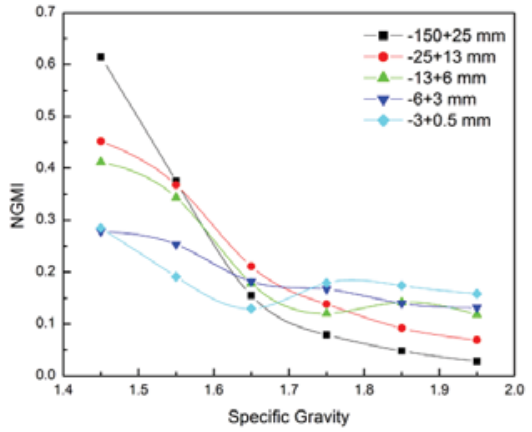

(a)

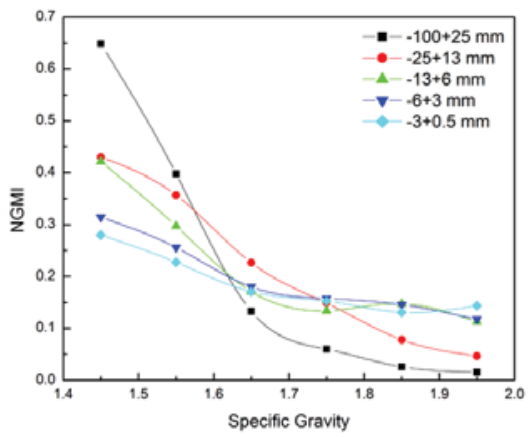

(b)

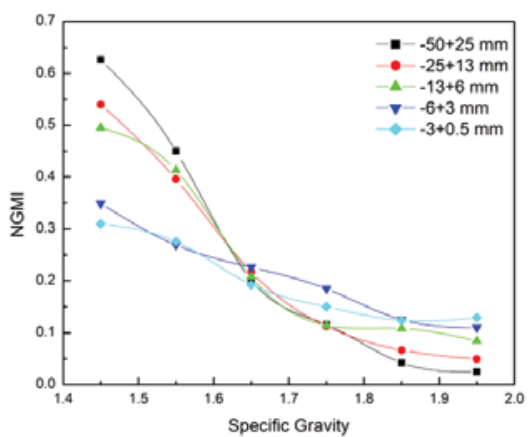

(c)

Figure 3-Plot of NGMI against specific gravity for different size fractions of run-of-mine coal crushed to (a) $150 \mathrm{~mm}$, (b) $100 \mathrm{~mm}$, (c) $50 \mathrm{~mm}$

with increasing specific gravity of separation. At lower specific gravity of separation the NGMI is highest for the coarser size fraction, whereas at higher specific gravity of separation it is lowest. This indicates that the coarser size fractions are more amenable towards washing at a higher specific gravity of separation and the finer size fractions are more amenable at a lower specific gravity of separation. Also, from Figure 3 it can be observed that the slope of the curve for the finer size fraction is less than that for the coarser size fraction. This indicates that the near-gravity material is distributed more-or-less uniformly in all the density classes for the finer size fractions. Therefore, the degree of difficulty involve in the separation process is almost same for finer size fractions, bur for coarser size fractions the difficulty is greater at lower specific gravity of separation due to the presence of more near-gravity material.

In order to clearly visualize the effect of crushing on near-gravity material distribution in different density classes, the NGMI was plotted against specific gravity, as shown in Figure 4 for the coarsest and finest size fractions when the three representative coal samples are crushed to $150 \mathrm{~mm}$, $100 \mathrm{~mm}$, and $50 \mathrm{~mm}$. It can be seen that the near-gravity material is distributed more uniformly in all the density classes for both the size fractions when the coal is crushed to $50 \mathrm{~mm}$. Therefore, the slopes of the curves for the coal crushed to $50 \mathrm{~mm}$ are less than the slopes of the curves for the coal crushed to $100 \mathrm{~mm}$ or $150 \mathrm{~mm}$. When the coal is crushed to $50 \mathrm{~mm}$, more finer particles are generated with various densities and these are equally distributed among all the density classes; whereas when the coal is crushed to 150 $\mathrm{mm}$ or $100 \mathrm{~mm}$ fewer finer particles are generated and most of the particles are larger. As a result they are not equally distributed in all the density classes.

The IW values for different size fractions of the coal were calculated for the three representative coal samples crushed to $150 \mathrm{~mm}, 100 \mathrm{~mm}$, and $50 \mathrm{~mm}$ and are shown in Table II. The IW values decrease with decreasing particle size for all three samples, which indicates the uniform distribution of the near-gravity material in all the density classes for smaller size fractions. A comparison between the coal crushed to 150 $\mathrm{mm}$ and coal crushed to $50 \mathrm{~mm}$ shows that the near-gravity material is distributed more uniformly between the density classes in the coal crushed to $50 \mathrm{~mm}$.

\section{Conclusions}

The preliminary results of this investigation indicate that finer crushing results in the uniform distribution of the neargravity material in all the density classes. This is probably because crushing generates a greater number of particles with different specific gravities. Therefore, the difficulty

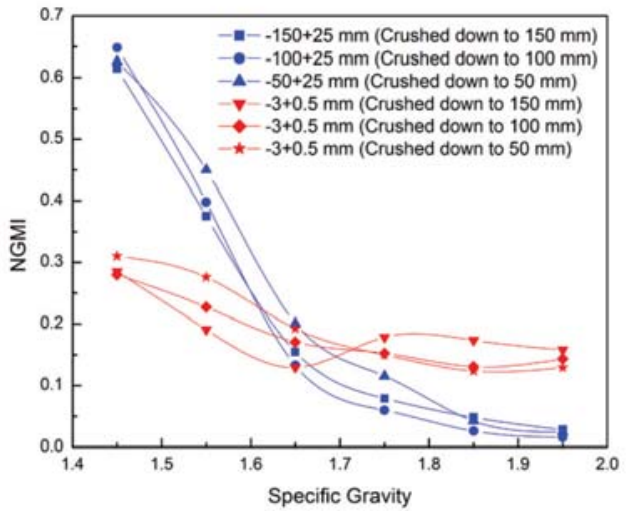

Figure 4-Plot of NGMI against specific gravity for $+25 \mathrm{~mm}$ and $-3+0.5 \mathrm{~mm}$ size fraction coal

Table /I

Index of washability for coal crushed to different sizes

\begin{tabular}{|l|c|c|c|}
\hline \multirow{2}{*}{ Size (mm) } & \multicolumn{3}{|c|}{$\begin{array}{c}\text { Index of washability (IW) for coal crushed } \\
\text { to different sizes }\end{array}$} \\
\cline { 2 - 4 } & $\mathbf{1 5 0} \mathbf{~ m m}$ & $\mathbf{1 0 0} \mathbf{~ m m}$ & $\mathbf{5 0 ~} \mathbf{~ m}$ \\
\hline+25 & 15.259 & 12.064 & 17.354 \\
$-25+13$ & 26.556 & 25.811 & 22.783 \\
$-13+6$ & 30.653 & 32.223 & 25.786 \\
$-6+3$ & 40.813 & 38.115 & 33.490 \\
$-3+0.5$ & 42.393 & 42.603 & 38.138 \\
\hline
\end{tabular}




\section{Effect of crushing on near-gravity material distribution in different size fractions}

involved in beneficiating finely crushed coal remains almost the same for all specific gravities of separation. With coarser crushing, more near-gravity material is present in the lower density class than the higher density classes. Beneficiation therefore becomes more difficult at a lower specific gravity of separation. However, this is a case-specific situation and it depends on the nature of the mineral distribution in the runof-mine coal.

\section{Acknowledgements}

The authors express their sincere thanks to The Institution of Engineers (India) for funding the project (Project I.D. UG2014012). Sincere gratitude is also extended to the coal companies that provided the samples for this investigation. Finally, the authors express their thanks to all colleagues who directly or indirectly contributed to the successful completion of this study.

\section{References}

Corriveau, M.P. and SchapiRo, N. 1979. Projecting data from samples. Coal Preparation. 4th edn. Leonard, J.W. (ed.). American Institute of Mining, Metallurgical, and Petroleum Engineers Inc., New York. Ch. 4, pp. 4-27.
GovindARAJAN, B. and RAo, T.C. 1994. Indexing the washability characteristics of coal. International Journal of Mineral Processing, vol. 42. pp. 285-293.

Indian Standard. IS: 1350, (Part I): 1984. Methods of Test for Coal and Coke. (Reaffirmed 2001).

Indian STANDARD. IS: 436, (Part I/Sec 1): 1964. Methods of Sampling of Coal and Coke. (Revised 1981).

INDIAN STANDARD. IS: 13810: 1993. Code of Practice for Sink-and-float Analysis of Coal.

MAJUMder, A.K. and BARNWAL, J.P. 2004. Development of a new coal washability index. Minerals Engineering, vol. 17. pp. 93-96.

Mitchell, D.R. and Charmbury, H.B. 1963. Cleaning and Preparation. Chemistry of Coal Utilization. Supplementary Volume. Lowry, H.H. (ed.). Wiley, New York. pp. 312-319.

OsBorne, D.G. 1988. Coal Preparation Technology, Vol. I. Graham and Trotman, London. pp. 179-188.

SALAmA, A.I.A. 1989. Theoretical aspects of parallel coal processing circuits. International Journal of Mineral Processing, vol. 27. pp. 171-188.

SALAmA, A.I.A. and MiкнAiL, M.W. 1993. Balancing of raw washability data utilizing the least squares approach. Coal Preparation, vol. 13. pp. 85-96.

SARKAr, G.G., Bose, R.N., Mitra, S.K., and Lahiri, A. 1962. An index for the comparison and correlation of washability characteristics of coal. IV Coal Preparation Congress, Harrogate. Paper E4.

SARKAR, G G., DAS, H.P., and GHoSE, A. 1977. Sedimentation patterns: do they offer clues to coal quality? World Coal. pp. 10-13. 